\title{
Analyzing the Effect of Vaping Use in Teens: A Literature Review and Proposed Solutions
}

\author{
Damilola Ajayi ${ }^{1}$, Bruce Fuchs ${ }^{1}$, and Dana Reiss ${ }^{1}$ \\ 'University of Maryland, College Park, MD, USA \\ DOI: https://doi.org/10.47611/jsr.v10i1.1109
}

\section{$\underline{\text { ABSTRACT }}$}

Teenage vaping is a major public health issue that is emerging globally. This review article examines what influences teens to use electronic cigarettes (e-cigarettes) and vapes, including families, communities, social media, and brand marketing. Nicotine, a common component of e-cigarettes and vapes, is addictive, and teens who are using these products are starting to report negative health outcomes. After a review of the current literature, three recommendations/solutions are proposed to combat this public health issue. As teen e-cigarette use is on the rise, it is clear that the FDA and the federal government need to enforce tougher regulations on e-cigarette and vaping brands, teens need to be properly educated about these products that are on the market, and advertisements for these products need to be regulated.

\section{Introduction}

Cigarette smoking has had a significant impact on populations for a considerable amount of time. E-cigarettes were created as an alternative to cigarettes over a decade ago. E-cigarettes are an electronic device that works by heating a liquid that produces small airborne particles (CDC, 2020a). They come with many different flavors that have been marketed as being a safe alternative to smoking cigarettes and, as a result, have become popular amongst young people, such as middle and high school aged children. The terms "vaping" and "JUULing" also refer to the act of using an e-cigarette (CDC, 2020a). There is evidence linking smoking to lung cancer through data over the years. There are warnings about e-cigarettes and health hazards as well, but there is no solid data that is similar to cigarettes and their harm.

For many people the major concern is that vaping is on the rise, especially among adolescents. It was reported that from 2011-2015, vaping amongst high school students increased from 1.5\% to 16\% (Singh et al., 2016). The troubling part about this increase is that e-cigarettes are harming people in what seems to be a shorter period of time than cigarettes. The Center for Disease Control and Prevention (CDC), Food and Drug Administration (FDA), and many state and local health departments are investigating lung injuries resulting from vaping and e-cigarette use otherwise known as e-cigarette or vaping product use-associated lung injury (EVALI) (CDC, 2020b). Some alarming numbers have been gathered by the CDC recently about EVALI cases. According to the CDC, 2,711 EVALI cases/deaths have been reported by all 50 states, Washington, D.C, Puerto Rico, and the U.S. Virgin Islands and 60 deaths have been confirmed in 27 states and Washington, D.C. (CDC, 2020b). This data is as of January 21, 2020 and changes as time goes on.

As it concerns the younger population, e-cigarettes have been deemed unsafe for teens (CDC, 2020a). Just like cigarettes, e-cigarettes contain nicotine, which is extremely addictive and can change the chemical composition of brains that are still developing (CDC, 2020a). What makes the sale of e-cigarettes to teenagers alarming is that they may not know what they are putting into their bodies. The CDC recently did a study that produced alarming results about the truth of e-cigarettes. It was reported that $99 \%$ of e-cigarettes sold in the United States (US) were found to 
contain nicotine even though some packaging labels did not include nicotine as being an active ingredient or said $0 \%$ nicotine was included (which was a false statement) (CDC, 2020a). There is also concern about what vaping may be doing to teens' lungs.

As previously mentioned, e-cigarettes come in many different flavors. However, many of the chemicals used to create flavors that our digestive systems can process cannot be processed by our lungs, so some e-cigarette flavors are also affecting teenagers' lungs (CDC, 2020a). Many teens like the idea of e-cigarettes because they are marketed differently than cigarettes. Cigarettes do not have fruity or candy flavors, but e-cigarettes do. Many teens have reported that they vape because they can pick from a variety of flavors to smoke (CDC, 2020a). Just because these devices have a flavor aspect to them, does not mean they are better or safer than cigarettes for anyone, let alone teens.

E-cigarettes have also been seen to do things that cigarettes would not necessarily do. There have been reports that some vapes have malfunctioned, which caused fires and explosions, resulting in serious injuries (CDC, 2020a). There also have been reports that children and adults were unfortunately poisoned from either swallowing, breathing, or absorbing vaping liquid by way of their skin or eyes (CDC, 2020a). This could be attributable to many different reasons, but overall, it is another reason the $\mathrm{CDC}$ recommended that teens not use e-cigarettes.

There is a cause for concern not only because of the effects that vaping can have, but also because of the number of teens who seem to be regularly using e-cigarettes. In 2019, more than one in four high school students reported that they vaped in the last month (CDC, 2019). This is troubling because that means more than $25 \%$ of high school students face potential lung injuries or other serious health consequences because of their use of e-cigarettes. This number may also continue to rise because of peer influence and lack of education, which would continue to exacerbate this public health issue among teens (Chao et al., 2019 \& Lippert et al., 2019).

The e-cigarette market is a billion-dollar industry that spends a large amount of money on advertising. Teens are also heavily exposed to e-cigarettes via advertising, which is also a concern. We know that the purpose of the ads is to inform its viewers of a product and ultimately entice them to buy or partake in the product that is being mentioned. Ads are persuasive and can pull their viewers in, so when individuals who are easily influenced, such as high school teens, view one, they are likely to go out and buy one or try one from friends (Nicksic et al., 2017). A report stated that seven in ten teens are exposed to ads that are marketing e-cigarettes (National Institute on Drug Abuse, 2016). Among high school teens, $56.3 \%$ see ads in stores, $42.9 \%$ see ads on the internet, $38.4 \%$ see ads on TV and in movies, and 34.6\% see ads in newspapers and magazines (National Institute on Drug Abuse, 2016). Since a high percentage of teens are finding out about e-cigarettes from ads, it is likely they have misconceptions about what is in these products.

When teens were asked what they thought were in e-cigarettes, a high percentage incorrectly reported these substances. An overwhelming $66 \%$ of teens thought there was only flavoring in e-cigarettes, while $13.7 \%$ reported that they did not know, and $13.2 \%$ thought nicotine was in them. While it is correct that most e-cigarettes contain nicotine, a relatively low percentage of people reported that correctly (National Institute on Drug Abuse, 2016). Also, $5.8 \%$ believed they contained marijuana (National Institute on Drug Abuse, 2016). Most of the information gathered thus far has been about teens' use of e-cigarettes in the United States; however, it is important to understand that teen vaping also occurs globally.

Teen vaping in Poland is similar to the use reported in the US. In a Polish study conducted in 2016 that consisted of 3,552 adolescents from ages 13-19, nearly $22 \%$ of participants reported having smoked an e-cigarette before and $27 \%$ reported that they had smoked in the last month (Kaleta et al., 2016). Males and females both reported having smoked before at the rate of $22 \%$, while current e-cigarettes use at the time among males and females was found to be $32 \%$ and $21 \%$, respectively (Kaleta et al., 2016). If teens are vaping not only in the US but in Poland, it is likely that they are vaping in many more countries. This is a global public health issue that has the potential to negatively impact many teens' health early in their lives. Thus far, many studies have described e-cigarettes as having many negative health effects on people, especially teens.

Based on the existing literature so far, it is clear that the components of e-cigarettes are causing lung damage as well as other health issues to people using them. We also know that marketing of e-cigarettes is driving many more 
people, especially teens, to use them which is increasing the prevalence of the problem. Nicotine is addictive, which explains why many people who smoke regular cigarettes have a hard time quitting. Since nicotine is also found in ecigarettes, existing literature suggests that teens who are regularly vaping may also be addicted. Many studies outline that teens should avoid the use of e-cigarettes. It is important to understand factors that impact teens' use of e-cigarettes, along with the negative health outcomes, to appropriately address the health and safety of teens going forward. The purpose of this paper is to examine the prevalence and negative health issues of teens in the US who are vaping. This paper will begin by examining the factors that influence teens to vape. Next it will discuss how marketing directed at teens is driving the use of e-cigarettes. Then it will discuss how nicotine is addictive. Most importantly, this paper will provide more detail about how exactly vaping is hurting teens, such as the types of lung injuries that people have reported. E-cigarettes are still relatively new compared to conventional cigarettes, so the long-term effects are not completely understood. Then a thesis statement will be presented and lastly, suggestions of possible interventions will be proposed to help address this public health issue.

\section{Literature Review}

\section{Methods}

Literature was selected by using search engines and key words to only include information related to the four main topics of the literature review. Google Scholar and Academic Search Complete were used to find the literature used in this review. Additional sources were found through work that was cited in literature from these search engines. Some of the keywords used in the search engines included "teen vaping social influence", "teen nicotine use", "regulating e-cigarettes", and "teen vaping marketing" just to name a few. A total of 29 articles were used in this review, and the articles that were chosen to be used in this review met the requirements of discussing adolescents, negative health outcomes as a result to vaping, the addictive nature of nicotine, and information related to brand marketing.

\section{Teen Influence}

Social influence of e-cigarette use from friend groups and the general population has been seen to affect "smoking behaviors" (Chao et al., 2019). "Smoking behaviors" refers to smoking regular cigarettes, e-cigarettes, or both. The prevalence of e-cigarette use has increased and is thought to likely be the explanation for how communities and individuals view vaping (Chao et al., 2019). In a study done of 3,000 high school students using data from the 2011-2014 National Youth Tobacco Survey, a decision model was created for high school students (see Figure 1) (Chao et al., 2019). Each participant reported whether they smoked or not, which was based on the utility calculation that was summed up by their own marginal utility derived from their smoking status, and the utility under social influence from friends in their circle and from the general population. Peer influence was accounted for by referring to the number of people who smoked in their close group of friends, while societal influence was accounted for based on the prevalence of different smoking types in the United States. Teens are influenced depending on how open they are to different types of smoking (Chao et al., 2019). After the behavioral study, the participants and their network of friends' behavioral status were revised and put back into the model to reshape a revised social influence environment, and the cycle was repeated. When a validity check was done on the model by comparing the actual trend of teen smoking prevalence, the researchers changed some of the essential parameters that related to teens' susceptibility to social influence to determine how the prevalence rates of the different types of smoking affect each other through teen's behavioral choices. 


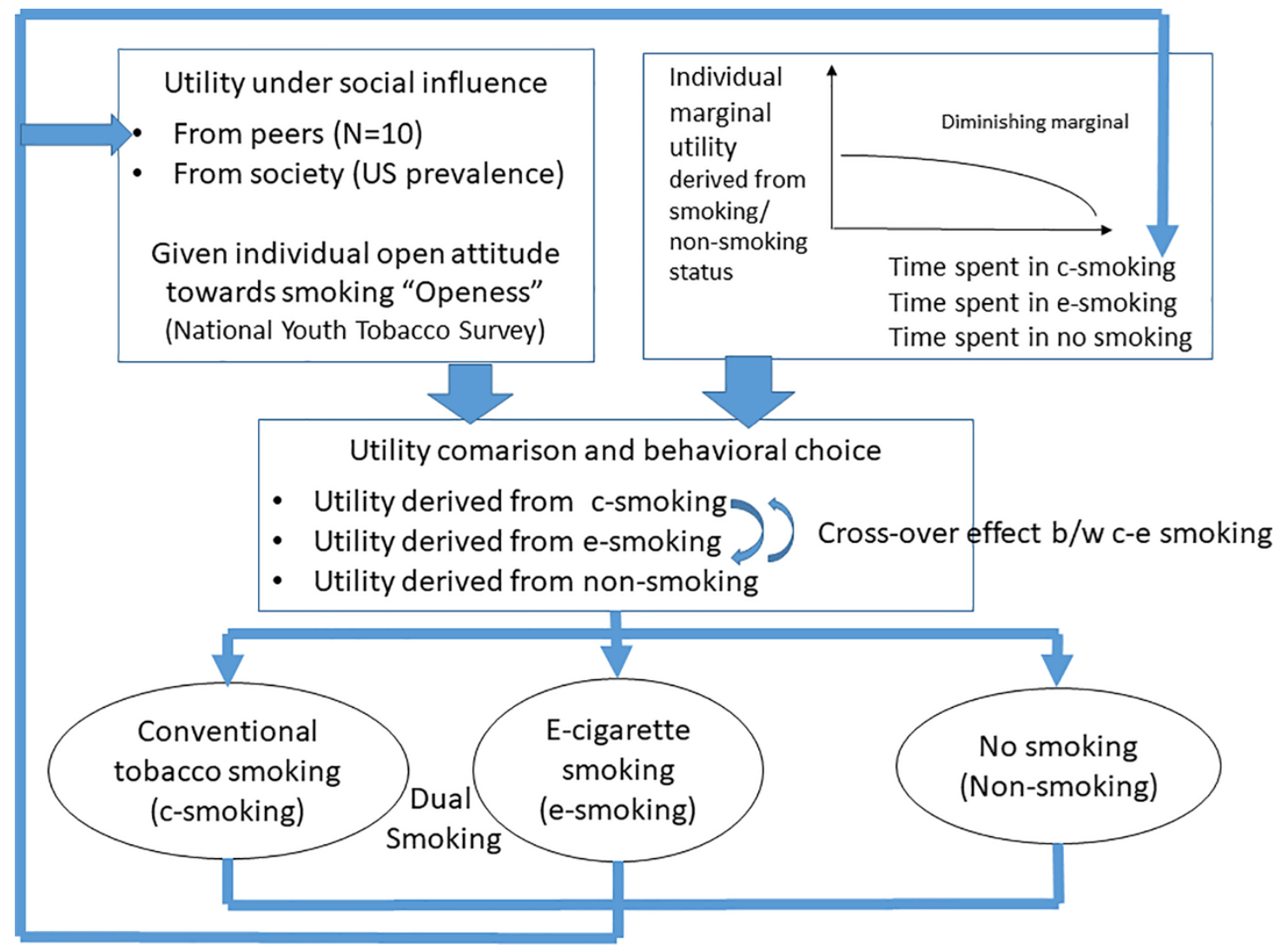

Figure 1. Cycle of behavioral choice for smoking status and types of smoking (Chao et al., 2019). Figure is from an open access article distributed under the terms of the Creative Commons Attribution License, which allows unrestricted use, distribution, and reproduction in any medium, provided the original author and source are credited.

The researchers in this study made the assumption that the participants chose whether to smoke or not based on their feelings and attitudes towards smoking, but they were also susceptible to being influenced by their peers and society as a whole (Chao et al., 2019). The study also included a simulation scenario that was based on the baseline model. The researchers explored multiple scenarios to examine how the participants' perspectives and social influence from peers influenced e-cigarette and regular cigarette use prevalence rates. This suggests that the decision model appropriately reported the prevalence trends in regular cigarettes, e-cigarettes, and dual smoking among high school students in the US (Chao et al., 2019). This is based on the 95\% confidence interval reported of prevalence rates (Chao et al., 2019). Chao et. al. (2019) also concluded that their simulation model “... provides insight into the mechanism by which young people's open attitudes to e-cigarette use and the prevailing popularity of e-cigarettes in their local networks and in the broader society can be strong drivers for rapid and massive prevalence increases" (p. 6).

Essentially, a correlation was found between peer and societal influence and e-cigarette prevalence. This is a problem because the more teens are around others who are vaping, the more likely they are to smoke. Many teens do not know what they are smoking when using e-cigarettes, which means that there is an education gap about these products that needs to be filled.

A weakness of the Chao et. al. (2019) article was that the researchers could not explain in enough detail how peers and communities could influence teens. Similarly, another recent study looked at the role of schools and peers in influencing students to use e-cigarettes (Lippert et al., 2019).

The study set out to answer two questions: "(1) How has the school clustering of vaping-a measure of the extent to which schools differ based on their prevalence of student e-cigarette use-changed between 2011 and 2017 as the population-wide prevalence of youth vaping increased? (2) How is the prevalence of school-level e-cigarette use 
associated with a student's odds of vaping net of key influences from one's family and peer group?" (Lippert et al., 2019, p. 1900). This study used the Ecological Systems Theory to investigate the question above. Based on the Ecological Systems Theory, young people are influenced by many different factors (Lippert et al., 2019). Anything from culture, public policy, and trends in families, peers, and schools can and often influences youth (Lippert et al., 2019). How exactly schools influence youth to use e-cigarettes is not entirely known; however, what is known is that in schools that have tolerant norms and high vaping rates, youth are more likely to believe that e-cigarettes do not cause any harm and are less addictive than conventional cigarettes (Lippert et al., 2019). In these schools that have tolerant norms and that do not educate youth on the facts about e-cigarettes, peers are very likely to obtain the resources they need for vaping (Lippert et al., 2019).

A cross-sectional study was completed in 2011, 2013, 2015, and 2017 using the National Youth Tobacco Survey (NYTS), which is a self-administered survey given to middle and high school students (Lippert et al., 2019). From 2011-2017, 65,067 students participated in the study (Lippert et al., 2019). Students were asked if they ever used e-cigarettes and if they had used them in the past 30 days (Lippert et al., 2019). Based on the NYTS, it was found that from 2011 to 2017, prevalence of lifetime e-cigarette use increased from 3.0\% to $20.8 \%$ and current e-cigarette usage increased from $1.0 \%$ to $7.8 \%$ (Lippert et al., 2019). Half of respondents in the study were male and the racial and ethnic demographics were widespread (Lippert et al., 2019). As the study progressed throughout the years, fewer participants reported living with a smoker, but second-hand smoke exposure from peers specifically increased between 2013 and 2015 (Lippert et al., 2019). Additionally, the study found that e-cigarette use was more likely among moderate/high use vaping schools compared to low-level ones (Lippert et al., 2019). Older students, males, and anyone exposed to secondhand smoke from a family member or peers, and students who previously used conventional cigarettes were also more likely to use e-cigarettes than other students (Lippert et al., 2019).

A correlation exists between adolescent development and the factors that are near to them such as peers, family, school and overall community. It was found that schools do play a role in the prevalence of vaping (Lippert et al., 2019). Based on the findings of the study, researchers believe that educating teens on the facts related to e-cigarettes and nicotine addiction is imperative for their overall health (Lippert et al., 2019).

Compared to the Chao et al. (2019) article, Lippert et al. (2019) comprised of more clarifying information. The researchers discussed many specific examples of what influences teens in general and then provided examples of what is influencing teens to vape. This study also had a larger sample size which means that the study was more inclusive. Lastly, the study discussed future steps that should take place in order to help reduce teen e-cigarette prevalence rates. Chao et al. (2019) did not explicitly state all of these details. It is clear from analyzing the article and research study that teens are often influenced to vape because people around them are using e-cigarettes. It also seems that social media drives teen influence toward e-cigarette use.

Social media is used to spread many different types of information to a high number of people instantly. If you are trying to influence people to be associated with your brand, social media is an easy and free way to connect with real people. A study looked into adolescents less than 18 years old who follow JUUL's official Twitter account and interact with their tweets (retweeting tweets) (Chu et al., 2018). Why should concern arise about JUUL's influence on social media? It is concerning because JUUL has taken over the e-cigarette market. JUUL is the fastest growing vaping company and is seen as the figurehead of the e-cigarette market (Chu et al., 2018). Online discussion boards have been found to provide information for adolescents on where they can obtain JUULs and their related products, and how teens can hide them so that they are not confiscated (Kavuluru et al., 2019). Without forums like these, many teens would not know where they could illegally obtain JUULs and other e-cigarettes, which means that these forums are promoting their use and influencing teens to vape. Many posts on social media as well as the news are reporting that JUULs and other e-cigarettes are being used in many high schools (as well as elementary and middle schools) (Allem et al, 2018). A large number of teens are being exposed to information such as ads and tweets from their friends referencing information related to vaping, which is influencing their knowledge and increasing the risk that they will vape (Soneji, et al., 2018). 
In Chu et al. (2018), researchers investigated adolescents less than 18 years old who follow JUUL's official Twitter account and interact with their tweets. Researchers wanted to analyze if teens were following JUUL's Twitter, how often they were sharing their posts by retweeting JUUL's tweets, and if there was a pattern of how teens share information about JUULs (Chu et al., 2018). The study collected all of JUUL's tweets from February 2017 to January 2018 (Chu et al., 2018). The number of tweets collected were 3,329, and these tweets were retweeted 1,124 times by 721 different people (Chu et al., 2018). This may not seem like a lot; however, if someone retweets a tweet and they have thousands of followers, there is a high chance that many of their followers will view that tweet. Coders analyzed the profiles of the 721 people who retweeted JUUL's tweets to figure out what age the users were (Chu et al., 2018).

Coders found that $25 \%$ of the users who interacted with the tweets were adolescents. Out of the 1,124 retweets, $9 \%$ of retweets came from teens retweeting a tweet from another teen and $12 \%$ of retweets came from teens retweeting a tweet from an adult (Chu et al., 2018). From the results, researchers found that teens are indeed following JUUL's twitter account and their tweets are also being retweeted by teens (Chu et al., 2018). This is an issue because social media is influencing teens to either start using e-cigarettes or to continue to use them. A strength of this article/study is that it picked the leading brand of e-cigarettes to analyze the influence of its products on social media among teens. It showed exactly how information often spreads to teens. It is clear that communities, peers, family, and social media plays a major role in teen e-cigarette use and knowledge. It is also safe to say that e-cigarette prevalence is higher due to communities, peers, family, and social media. Another major factor is how brands market their products.

\section{Marketing to Teens}

It is safe to say that teens are finding out about e-cigarettes in multiple ways every day. However, it seems that an overwhelming number of teens are being influenced by e-cigarettes through marketing. Since 2011, spending on ecigarette marketing has quickly increased (see Figure 2) (CDC, 2017). Approximately $69 \%$ of middle and high school students were exposed to e-cigarette marketing via the internet, movies, magazines, and retail stores just to name a few (CDC, 2017). E-cigarette prevalence has been steadily increasing among high school students for several years, and being exposed to such ads may be contributing to the increasing prevalence rates (CDC, 2017). Teens are being exposed to e-cigarette ads at an alarming rate, and the problem with this is that teens are easily influenced, which means the chance of teens using e-cigarettes becomes greater just from seeing ads (CDC, 2017).

\section{E-cigarette use among youth is rising as e-cigarette advertising grows}

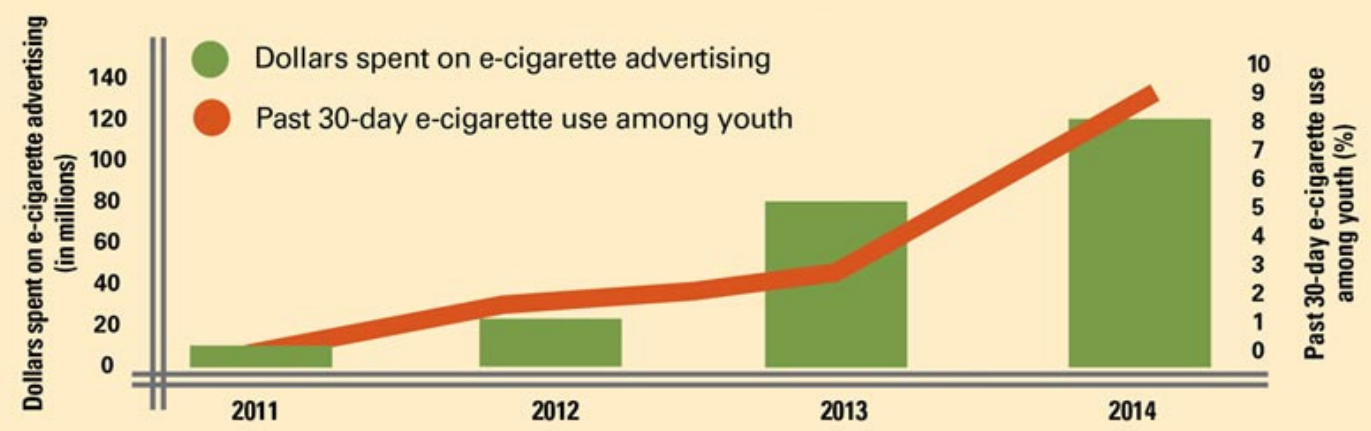

SOURCE: National Youth Tobacco Survey, 2011-2014; Kim et al (2014). Truth Initiative (2015).

Figure 2. Graph showing e-cigarette use among youth as advertising grows (CDC, 2017). This image was created by the $\mathrm{CDC}$; therefore, it is of public domain. 
As seen in Figure 2, from 2011 to 2014, e-cigarette advertisement spending has increased from $\$ 6.4$ million to $\$ 115$ million (CDC, 2017). This sharp increase in marketing of e-cigarettes and as well as the subsequent prevalence of e-cigarette use among teens is making many people talk about what can be done to fix this problem.

Currently, the federal government is attempting to prevent teens from using tobacco products by supporting state prevention and control programs, providing information to the public about the health effects of vaping, and creating regulations on e-cigarettes just to name a few (CDC, 2017). Healthcare providers should ask teens about their knowledge of e-cigarettes, whether or not they use them, and counsel them on the dangers of these products (CDC, 2017). Parents should monitor what media their kids may be viewing, determine whether the content they are viewing is appropriate for them, and sit down with their children to discuss the harm in vaping (CDC, 2017). There is a lot of information (true and false) about e-cigarettes and their effects on teenager's health; however, research does seem to support that marketing has an impact on vaping prevalence.

Researchers conducted a study that aimed to observe the impact of e-cigarette marketing on e-cigarette use among youth (Nicksic et al., 2017). From 2011 to 2012, the amount of money spent on advertising e-cigarettes tripled, and the majority of ads were placed on television (Nicksic et al., 2017). Also, from 2011 to 2012, ads on the radio quadrupled and from 2011 to 2013, and e-cigarette ads were reaching 24 million children who were around the ages of 10-19 (Nicksic et al., 2017). In 2014, 70\% of adolescents in the United States saw e-cigarette ads on TV, the internet, and in stores just to name a few (Nicksic et al., 2017). The effect of advertising e-cigarettes to adolescents is a relatively new topic which means there are not many articles that discuss this information (Nicksic et al., 2017). However, in some studies that have been done, a trend was observed between high school students remembering an e-cigarette ad they had seen either on tv, the internet, in retail stores, etc. and increased e-cigarette use (Nicksic et al., 2017).

This recent study used cross-sectional analyses and longitudinal data. Data from this study were taken from the Texas Adolescent Tobacco Advertising and Marketing Surveillance System (TATAMS), which is a study that includes middle and high school students from the largest cities in Texas (Nicksic et al., 2017). This study was conducted in Texas and may provide a lot of insight to e-cigarette use because advertisers in Texas spend more money on tobacco advertising than any other state (Nicksic et al., 2017). The purpose of the study was to see whether remembering e-cigarette ads teens heard or saw in various places affected their past or present use of e-cigarettes, whether they viewed e-cigarettes as being dangerous, and how likely they were to use e-cigarettes (Nicksic et al., 2017).

The TATAMS study followed students aged 12-17 from 79 middle and high schools in five counties of the four most populated cities in Texas (Nicksic et al., 2017). From 2014-2015, baseline data and then six-month followup data were taken from the same group (Nicksic et al., 2017). The sample included 461,069 adolescents; however, 3,907 students completed the baseline survey and 2,488 out of the 3,907 students completed the follow-up survey six months later (Nicksic et al., 2017). Among students who completed the baseline and follow-up survey, $47.2 \%$ of teens remembered seeing an e-cigarette ad on TV, $63 \%$ in retail stores, $43.5 \%$ on the internet, and $23.7 \%$ remembered hearing an e-cigarette ad on the radio (Nicksic et al., 2017). Among teens who remembered e-cigarette ads on TV at baseline, a perception of no harm from e-cigarettes at follow-up was 1.49 times higher when compared to those who did not remember the ads (Nicksic et al., 2017). This means that when teens view ads and remember them, they are more likely to believe that e-cigarettes are not harmful. There potentially is a correlation between recalling these ads and thinking whether they are harmful or not which would ultimately influence e-cigarette usage.

Among teens who remembered ads from different outlets (TV, internet, retail stores, etc.), the chances of being influenced to use e-cigarettes was 1.76-2.79 times higher than teens who did not remember any ads (Nicksic et al., 2017). This is important because no matter the form of e-cigarette ads, a negative effect is being observed: teens are being influenced to use e-cigarettes. Many teens do not think there is anything wrong with these e-cigarettes especially because of the heavy advertising they are seeing everywhere. Ads for e-cigarettes can say anything in them, which seems to be driving teens to use them. Since e-cigarettes are considered to still be relatively new, many people do not know about all of the negatives associated with them, which also contribute to teens being easily influenced by companies' marketing. A strength of this study is that it looked at Texas's effect on influencing teens with e-cigarette ads. Since Texas spends the most on marketing e-cigarettes out of all 50 states, the location was ideal for the 
researchers to test the hypothesis that ads influence teens to use e-cigarettes. One weakness of this study is that the variables are self-reported (Nicksic et al., 2017). With self-reported variables comes the possibility of bias because asking people whether or not they recall memories can be distorted and not all memories are retained (Nicksic et al., 2017). Overall, however, this is a strong study that contributes to the growing number of studies being done on the effect of advertising on teens. The results seem correct as other studies done on e-cigarette advertising's effect on ecigarette usage among teens have found similar results.

In a randomized control trial done in 2014, the effect of viewing e-cigarette ads on TV was studied (Farrelly et al., 2015). Researchers described that in recent years, exposure to e-cigarette advertisements as well as e-cigarette use had increased even though concerns were raised about how safe the products are (Farrelly et al., 2015). This study was also the first of its kind to explore the effects of e-cigarette ads on teens age 13-17 using a randomized control trial (Farrelly et al., 2015). The study's purpose was to see if viewing e-cigarette ads on TV influenced teens to vape in the future and how the participants viewed e-cigarettes (Farrelly et al., 2015).

In 2014, researchers had approximately 5,000 adolescents (between the ages of 13-17) fill out an online survey (Farrelly et al., 2015). Of the approximately 5,000 participants, 26\% had used e-cigarettes previously and 74\% had not tried them (Farrelly et al., 2015). The adolescents in this study were exposed to approximately 40 e-cigarette ads, and 13 ads were used in a pretest study to examine reactions (Farrelly et al., 2015). The 13 ads that were used in the pretest study were chosen because they were either aired frequently or to a large number of people (Farrelly et al., 2015). Some of the main messages used in these ads were that e-cigarettes were safer to use than regular cigarettes, ecigarettes could be used in places regular cigarettes could not (i.e., a school), vapor coming from e-cigarettes does not negatively affect surrounding people, and using e-cigarettes would make people feel more independent and free (Farrelly et al., 2015). Participants were placed into two groups: the treatment group (exposed to e-cigarette ads) and the control group (not exposed e-cigarette ads) (Farrelly et al., 2015).

Teens in the treatment group reported a higher chance of using e-cigarettes in the future than the control group (Farrelly et al., 2015). Students who currently smoked conventional cigarettes showed a high correlation for future e-cigarette use (Farrelly et al., 2015). Teens who reported using conventional cigarettes were found to be more likely to use e-cigarettes in the future, and teens in the treatment group were also more likely to view e-cigarettes as safe alternative to conventional cigarettes compared to those in the control group (Farrelly et al., 2015).

The effects of e-cigarette advertising on TV is truly concerning, especially when large amounts of money are used to promote these products. Approximately $\$ 80$ million was spent on advertising of e-cigarettes in 2013, which means many teens were undoubtedly exposed to messages that promoted vaping (Farrelly et al., 2015). This study proved that exposing teens who had never smoked e-cigarettes to even a small number of e-cigarette ads correlated with favorable attitudes towards vaping and even encouraged them to try it in the future (Farrelly et al., 2015). The treatment group had positive views on e-cigarettes that were 50\% higher than the control group (Farrelly et al., 2015). One limitation of the study is that it did not monitor the participants e-cigarette use longitudinally, which means the researchers could not tell if the intent to use e-cigarettes in the future actually led to increased prevalence of e-cigarette use (Farrelly et al., 2015).

\section{Addictive Nature of Nicotine}

Nicotine has been said to be addictive for years and is a major reason why it is hard for people who smoke regular cigarettes to quit. Nicotine is also a component of e-cigarettes, providing one explanation for why e-cigarette prevalence is high and continues to rise. But what exactly makes nicotine addictive? Consuming nicotine regularly causes dopamine to be released in the brain (The NIDA Blog Team, 2019). Dopamine being released turns on the pleasure circuit of the brain, and this release tells the body to do the same behavior over and over and, in this case, the behavior would be using nicotine, which is in e-cigarettes (The NIDA Blog Team, 2019). Dopamine is released after each puff of a cigarette or vape is taken in, so if someone is taking in 100 puffs a day, the brain is being told to continuously use nicotine which is what causes an addiction (The NIDA Blog Team, 2019). 
During adolescence, the parts of the brain regulating emotions and cognition are still developing (Yuan et al., 2015). Long term exposure to nicotine during adolescence has major repercussions, such as diminished cognitive function, which could cause teens to later become less focused and more impulsive (Counotte et al., 2009, 2011; Trauth et al., 2000). Teen dependence on nicotine has been looked at in various studies.

In a cross-sectional survey conducted among adolescent smokers, the modified Fagerstrom Tolerance Questionnaire (mFTQ), risk factors of nicotine addiction, and demographics was used in four countries: Russia, Spain, Thailand, and the US (Prokhorov et al., 2017). The risk factors that were looked at were alcohol use, marijuana use, number of cigarettes smoked previously, and how old participants were when they tried their first cigarette (Prokhorov et al., 2017). This study tested how valid and reliable the mFTQ survey was in measuring potential nicotine dependence on adolescent smokers (Prokhorov et al., 2017).

Russia, Spain, Thailand, and the US were chosen to be a part of this study due to their participation in a tobacco use cessation and prevention program for teens called Project EX (Prokhorov et al., 2017). Besides the fact the above four countries were involved in international translation of the previously mentioned project, these countries also included students who were all smokers at baseline, and they all had sample sizes more than 100 (Prokhorov et al., 2017). Participants were eligible to be given the mFTQ survey if they were in between the age of 12-19 and had at least one cigarette in the past month (Prokhorov et al., 2017). In the US, students from 12 high schools in California were recruited for the survey (Prokhorov et al., 2017).

The $\mathrm{mFTQ}$ was used to look at nicotine dependence, and seven questions were asked in the survey relating to daily cigarette use. Among the 1097 participants in the study, 410 were smokers under the age of 17 and mainly identified as male (Prokhorov et al., 2017, p.692). The study found that the mFTQ is a valid and reliable test in determining nicotine dependence in these countries (Prokhorov et al., 2017). Testing done in this study represents aspects relating to teens being addicted to nicotine (Prokhorov et al., 2017). This study did a good job at looking at adolescents from four nations across the world. This is a strength because it reinforced the reliability and validity of the mFTQ survey.

\section{Vaping's Negative Impact on Health}

E-cigarettes have been marketed as and said to be a "safe-alternative" to conventional cigarettes (Maloney and Capella, 2015, p. 130). Though many teens may think that e-cigarettes are harmless, more and more data are showing otherwise (Raloff and Mole, 2015). The chemicals found in e-cigarettes can damage lung tissue causing inflammation, which makes it harder to breathe (Raloff and Mole, 2015).

Due to the increased recognition of the effect that e-cigarettes have on the respiratory system, more researchers have started analyzing their impact, especially in teens. A review studied the effects of vaping among teens who had various acute respiratory illnesses that were exposed to vaporized cannabis/nicotine (Thakar et al., 2020). The purpose of this review was to analyze the radiological imaging of lung injuries that were a result of vaping (Thakar et al., 2020).

Using a pulmonary database, researchers identified teens who were diagnosed with lung injuries from ecigarettes at a pediatric hospital over a 3-month time frame (Thakar et al., 2020). The teens studied were diagnosed based on three factors: their history of e-cigarettes use, radiological images that showed a lung injury, and ruling out other potential causes by looking at the patient's history and doing a thorough investigation (Thakar et al., 2020). Researchers also studied the teens medical records, and clinical findings included dyspnea (shortness of breath), hypoxia (not enough oxygen in the tissues), chest and abdominal pain, etc. (Thakar et al., 2020).

The final review group included 10 boys and two girls between ages 16-18 (Thakar et al., 2020). Out of the final group, nine of the teens experienced dyspnea, 11 experienced nausea/vomiting, nine had a cough, six had chest pain, and five had abdominal pain (Thakar et al., 2020). A test of pulmonary function was assessed in 10 out of 12 of the teens (Thakar et al., 2020). Three teens had diminished diffusion capacity, while two had an obstructive breathing 
pattern, two had a restrictive breathing pattern, and two had an obstructive/restrictive (mixed) breathing pattern (Thakar et al., 2020).

All but two of the teens in the final group used nicotine (Thakar et al., 2020). Overall, the use of e-cigarettes suggests a correlation with the pulmonary diseases the teens experienced. It is safe to say that teens should avoid ecigarettes to avoid respiratory issues in the future. This small group of teens in this review suggests that nicotine and e-cigarettes negatively affect teens' health; however, a limitation of the review is that it only looked at a small group for a small period of time. What would the data show if a larger group was followed for a longer period of time? A strength is that the review included teens who vaped at different times prior to their admission to the hospital (one vaped a month prior, another two months prior, a week prior, etc.) (Thakar et al., 2020).

A similar study evaluated chest radiographic and CT findings of lung injuries from vaping in adolescents (Artunduaga et al., 2020). Participants in the study were pulled from a computer database at Children's Medical Hospital in Dallas (Artunduaga et al., 2020). Researchers searched for teens up to 18 years old from December 2018 to December 2019 (Artunduaga et al., 2020). Patients in the study met the inclusion criteria, which included meeting the criteria set forth by the CDC for EVALI (see Table 1) and had imaging available when the study began (Artunduaga et al., 2020).

Table 1. "Summary of CDC Surveillance Case Criteria Definition for Severe Pulmonary Disease Associated with Electronic Cigarette or Vaping Product Use-associated Lung Injury (EVALI)" Permission was given to reproduce this table from the journal titled Radiology. Artunduaga et al. Radiology 2020;295:430-438.

\section{Table 1: Summary of CDC Surveillance Case Criteria Defini- tion for Severe Pulmonary Disease Associated with Elec- tronic Cigarette or Vaping Product Use-associated Lung Injury (EVALI)}

1. Electronic (e-cigarette) use or dabbing (superheated aerosol inhalation of substances with high THC concentration) during the 90 days before symptom onset

2. Pulmonary opacities at chest radiography or ground-glass opacities at chest CT

3. Absence of pulmonary infection on initial work-up (after minimum criteria met) OR infection identified with culture, polymerase chain reaction, or minimum criteria not met (testing not performed), but the clinical team believes this infection is not the sole cause of the underlying lung injury.

4. No clinical evidence in the medical record of plausible alternative diagnoses (eg, cardiac, rheumatologic, or neoplastic process)

Note. $-\mathrm{CDC}=$ Centers for Disease Control and Prevention, $\mathrm{THC}=$ tetrahydrocannabinol. Source. - Reference 9 .

The cohort of teens in this study included seven males and seven females between the ages of 13-18 (Artunduaga et al., 2020). Teens in the cohort had used e-cigarettes or tetrahydrocannabinol concentration (THC) in the 90 days prior to developing any symptoms (Artunduaga et al., 2020). Symptoms that some or all of the teens experienced were shortness of breath, coughing, chest pain, dyspnea on exertion, labored breathing, vomiting, nausea, diarrhea, and abdominal pain (Artunduaga et al., 2020). The imaging findings from the chest radiographic and CT images showed abnormalities and changes in the lungs and was described as being damaged (Artunduaga et al., 2020).

The study found that teen e-cigarette use was associated with EVALI, which was confirmed via chest radiographic and CT imaging (Artunduaga et al., 2020). It is important to note that vitamin E acetate, which is found in ecigarettes with THC, has been linked to several deaths. The substance has been found in the lungs of patients with 
EVALI and not in healthy people (CDC, 2019b; Ellington et al., 2020). "When these potentially harmful chemical constituents are inhaled, it is plausible that acute lung injury may occur" (Artunduaga et al., 2020, p. 436).

The Artunduaga et al. (2020) study was similar to the Thakar et al. (2020) study in that the teens in both studies were affected by most of the same symptoms as a result of their e-cigarette use. Artunduaga et al. and Thakar et al. showed that e-cigarette use has negative health outcomes, especially in adolescents.

\section{Side Effects of E-cigarette Use}

E-cigarette use poses major threats to adolescents. The FDA analyzed e-cigarettes from two top brands and found that the vapes contained carcinogens and diethylene glycol, a component of antifreeze (Northwestern Medicine, 2019). Ingesting carcinogens and a component of anti-freeze is essentially consuming poison. Another negative of ingesting carcinogens is an increased risk of cancer (Shmerling, 2019). A particular cancer has not yet been identified as the type of cancer e-cigarette users are at risk of. Many vapes also contain diacetyl which is a food additive used to flavor these devices (Laucks \& Salzman, 2020). Diacetyl was previously seen to cause bronchiolitis obliterans organizing pneumonia (BOOP). (Laucks \& Salzman, 2020). While diacetyl is safe to eat, it may not be safe to vaporize the substance and it inhale it (Laucks \& Salzman, 2020).

Additionally, the use of e-cigarettes can result in breathing issues in smokers and non-smokers (Northwestern Medicine, 2019). As previously mentioned, a common substance in vapes is nicotine. Nicotine can cause high blood pressure, narrowing arteries and impeding blood flow to vital organs (Northwestern Medicine, 2019). These are just a few side effects as a result of a vaping. Adolescents should not be experiencing such side effects at young ages because their bodies are developing. Perhaps learning more about the recorded side effects of vaping will encourage young adults to reconsider using such products.

\section{Summary and Thesis Statement}

What is currently known based on literature is that teens are easily influenced by peers and social media to vape and that advertising is driving the market and influencing teens. It is also known that the addictive nature of nicotine, especially in teens is negatively impacting their health. Considering much of the information that has been found on the impact of e-cigarettes, especially in teens, it is important that permanent changes are put in place to decrease ecigarette prevalence and improve teen health. As previously stated, adolescents' bodies are still developing until the age of 25, which means that e-cigarettes can be very harmful, especially when they are in between the age of 13-19. Undoubtedly, we must find a way to make it difficult or impossible for teens to obtain e-cigarettes and their related products in order to preserve lives. To decrease teen e-cigarette prevalence, harsher penalties need to be enforced, we need to educate teens on the reality of e-cigarette use, which has proven to be dangerous, and we need to regulate advertisements.

\section{Recommendations/Solutions}

\section{Harsher Penalties from the FDA and Federal Government}

Teens seem to obtain e-cigarettes and their related products in a variety of ways. Whether they get these products from the store, family members, friends, or the black market, their access needs to be limited. How can this be done? We must start with making it difficult to obtain these products. In 2016, The FDA finalized a regulation that gave them authority over e-cigarettes (Truth Initiative, 2019). Its "Deeming Rule" regulation gave the FDA complete control over aspects of e-cigarettes such as manufacturing, advertising, sale, and distribution of e-cigarettes (Truth Initiative, 
2019). The FDA also included in their "Deeming regulation" that no new e-cigarette products could come out without being reviewed prior to being put on the market and authorization from the FDA (Truth Initiative, 2019).

Unfortunately, companies are not complying with parts of the Deeming regulation, such as pre-market review and authorization from the FDA (Truth Initiative, 2019). A good example of this dates back to October 2019 when the FDA sent a letter to the e-cigarette company Eonsmoke because they had over 100 products that came onto the market without FDA approval beforehand (Truth Initiative, 2019). This should be illegal, so much so that if broken, companies should face heavy fines and even jail time.

Stricter penalties need to be enacted because if companies are not following regulations set forth by the FDA and the FDA only sends these companies a letter saying they are not in compliance with their regulations, they will continue to put their unregulated products out on the market. The FDA needs to send a message to companies like Eonsmoke that makes it harder for them to not follow the regulations set forth by the FDA. Ultimately, this would make it very difficult for unregulated products to be on the market as well as harder for teens to obtain these products. The federal government in conjunction with the FDA needs to create harsher penalties.

\section{Education}

Much of the information teens receive about e-cigarettes does not come from reputable sources. Teens mainly find out about e-cigarettes from social media, peers, and advertisements. These sources do not give teens comprehensive information on e-cigarettes such as the nicotine content and health implications. Advertisements in particular aim to get its viewers to buy the products companies are trying to sell. We need to change the source of information regarding e-cigarettes so that teens are receiving correct information. Many schools provide education regarding health to their students, which means that health classes would be a good place to educate teens on e-cigarettes.

Schools should make prevention measures a top priority to help decrease prevalence rates (University of Colorado Denver, 2019). Health education programs that are focused on how teens can become addicted to nicotine as well as how e-cigarettes can negatively impact one's health would fill existing gaps of knowledge (University of Colorado Denver, 2019). Adam Lippert, PhD, an assistant professor in the sociology at the University of Colorado Denver says that, "...schools can be very effective at getting the message out that e-cigarette use is a serious risk to teen health. With the right resources, schools can be a strong partner in the fight against adolescent nicotine use" (University of Denver Colorado, 2019).

If schools are not already providing information to their students about e-cigarettes, the curriculum needs to be changed. With the proper tools, schools can decrease the prevalence rates. Providing teens with information from trusted sources such as the CDC would clarify the information that teens are learning through ads, social media, and their peers.

A potential limitation of educating teens in schools is that teens may not take the information they are being taught seriously. It might be helpful for advocates who have been personally affected by the use of e-cigarettes to talk to students in person or via a documentary. Students being educated by someone who was negatively impacted by ecigarette use may enhance their understanding of the severity of e-cigarettes.

To be clear, it is unknown if there are any rigorous studies that have investigated whether properly educating teens on the use of e-cigarettes will decrease e-cigarette prevalence. Due to there not being data on prior and post knowledge gain of adolescents after an educational intervention, it is only an idea that education would help decrease prevalence rates.

\section{Regulate Advertisements}

Research has proven that e-cigarette advertisements negatively influence teen vaping (Nicksic et al., 2017). TV commercials, radio ads, and retail ads are examples of the types of ads that need to be regulated by the federal government. Since e-cigarette ads are influencing teens to use e-cigarettes (Nicksic et al., 2017), perhaps increased regulations and 
monitoring would help decrease e-cigarette prevalence. Ads should be more transparent, and since the majority of ecigarettes contain nicotine in them, ads should include a message about the toxins and harmful chemicals that can be found in e-cigarettes. Ads should also include potential health risks that are associated with using e-cigarettes.

A potential limitation of regulating advertisements is that even regulated ads may still influence teens. There is no guarantee that with more transparent ads that teens will not be influenced to try the products. Advocates who have been affected by e-cigarette use at a young age could share their stories in the ads, which may encourage teens to avoid using e-cigarettes.

\section{Conclusion}

The overall goal of communities, parents, schools, and the federal government should be to decrease e-cigarette prevalence. We have seen how teens are influenced by many factors to vape, such as peers, social media, and advertisements. We have also seen that e-cigarettes have negative effects on people's bodies, especially teens. Potential nicotine addiction and respiratory issues stemming from lung injuries due to e-cigarette use should not be taken lightly. Focusing on primary, secondary, and tertiary prevention efforts will help decrease e-cigarette prevalence as well as promote overall health and well-being. Through harsher penalties from the FDA and federal government for companies that are not following the regulations, properly educating teens, and regulating advertisements, we can help teens and communities be less affected by e-cigarettes and save lives.

\section{Acknowledgments}

Special thanks to Dr. Dana Reiss, my instructor for the senior capstone course, and Dr. Bruce Fuchs, my mentor, for their support throughout my writing process. Thank you to the UMD Libraries' Open Access Publishing Fund for support.

\section{References}

Allem, J.-P., Dharmapuri, L., Unger, J. B., \& Cruz, T. B. (2018). Characterizing JUUL-related posts on Twitter. Drug and Alcohol Dependence, 190, 1-5. https://doi.org/10.1016/j.drugalcdep.2018.05.018

Artunduaga, M., Rao, D., Friedman, J., Kwon, J. K., Pfeifer, C. M., Dettori, A., ... Lee, E. Y. (2020). Pediatric Chest Radiographic and CT Findings of Electronic Cigarette or Vaping Product Use-associated Lung Injury (EVALI). Radiology, 192778. https://doi.org/10.1148/radiol.2020192778

CDC. (2017, March 23). E-cigarette Ads and Youth. Retrieved March 3, 2020, from https://www.cdc.gov/vitalsigns/ecigarette-ads/index.html

CDC. (2019a, December 10). Youth and Tobacco Use. Retrieved January 31, 2020, from https://www.cdc.gov/tobacco/data statistics/fact sheets/youth data/tobacco use/index.htm

CDC. (2019b, December 19). Outbreak of Lung Injury Associated with E-Cigarette Use, or Vaping.

https://www.cdc.gov/tobacco/basic+information/e-cigaretttes/severe-lung-disease.html.

CDC. (2020a, January 3). Quick Facts on the Risks of E-cigarettes for Young People. Retrieved January 30, 2020, from https://www.cdc.gov/tobacco/basic information/e-cigarettes/Quick-Facts-on-the-Risks-of-E-cigarettes-forKids-Teens-and-Young-Adults.html 
CDC. (2020b, January 28). Smoking and Tobacco Use; Electronic Cigarettes. Retrieved January 30, 2020, from https://www.cdc.gov/tobacco/basic information/e-cigarettes/severe-lung-disease.html

Chao, D., Hashimoto, H., \& Kondo, N. (2019). Social influence of e-cigarette smoking prevalence on smoking behaviours among high-school teenagers: Microsimulation experiments. PLOS ONE, 14(8), e0221557. https://doi.org/10.1371/journal.pone.0221557

Chu, K.-H., Colditz, J. B., Primack, B. A., Shensa, A., Allem, J.-P., Miller, E., ... Cruz, T. B. (2018). JUUL: Spreading Online and Offline. Journal of Adolescent Health, 63(5), 582-586. https://doi.org/10.1016/j.jadohealth.2018.08.002

Counotte, D. S., Goriounova, N. A., Li, K. W., Loos, M., Van Der Schors, R. C., Schetters, D., ... \& Spijker, S. (2011). Lasting synaptic changes underlie attention deficits caused by nicotine exposure during adolescence. Nature neuroscience, 14(4), 417.

Counotte, D. S., Spijker, S., Van de Burgwal, L. H., Hogenboom, F., Schoffelmeer, A. N., De Vries, T. J., ... \& Pattij, T. (2009). Long-lasting cognitive deficits resulting from adolescent nicotine exposure in rats. Neuropsychopharmacology, 34(2), 299-306.

Ellington, S., Salvatore, P. P., Ko, J., Danielson, M., Kim, L., Cyrus, A., .. Wallace, B. M. (2020). Update: Product, Substance-Use, and Demographic Characteristics of Hospitalized Patients in a Nationwide Outbreak of E-cigarette, or Vaping, Product Use-Associated Lung Injury — United States, August 2019-January 2020. MMWR. Morbidity and Mortality Weekly Report, 69(2), 44-49. doi: 10.15585/mmwr.mm6902e2

Farrelly, M. C., Duke, J. C., Crankshaw, E. C., Eggers, M. E., Lee, Y. O., Nonnemaker, J. M., ... Porter, L. (2015). A Randomized Trial of the Effect of E-cigarette TV Advertisements on Intentions to Use E-cigarettes. American Journal of Preventive Medicine, 49(5), 686-693. https://doi.org/10.1016/j.amepre.2015.05.010

Kaleta, D., Wojtysiak, P., \& Polańska, K. (2016). Use of electronic cigarettes among secondary and high school students from a socially disadvantaged rural area in Poland. BMC Public Health, 16(1), 1-10. https://doi.org/10.1186/s12889-016-3417-y

Kavuluru, R., Han, S., \& Hahn, E. J. (2019). On the popularity of the USB flash drive-shaped electronic cigarette Juul. Tobacco Control, 28(1), 110-112. https://doi.org/10.1136/tobaccocontrol-2018-054259

Laucks, P., \& Salzman, G. A. (2020). The Dangers of Vaping. Missouri medicine, 117(2), 159-164.

Lippert, A. M., Corsi, D. J., \& Venechuk, G. E. (2019). Schools Influence Adolescent E-Cigarette use, but when? Examining the Interdependent Association between School Context and Teen Vaping over time. Journal of Youth and Adolescence, 48(10), 1899-1911. https://doi.org/10.1007/s10964-019-01106-y

Maloney, E. K., \& Cappella, J. N. (2016). Does Vaping in E-Cigarette Advertisements Affect Tobacco Smoking Urge, Intentions, and Perceptions in Daily, Intermittent, and Former Smokers? Health Communication, 31(1), 129138. https://doi.org/10.1080/10410236.2014.993496

National Institute on Drug Abuse. (2016, February 11). Teens and E-cigarettes. Retrieved from https://www.drugabuse.gov/related-topics/trends-statistics/infographics/teens-e-cigarettes 
Nicksic, N. E., Harrell, M. B., Pérez, A., Pasch, K. E., \& Perry, C. L. (2017). Recall of E-cigarette Advertisements and Adolescent E-cigarette Use. Tobacco Regulatory Science, 3(2), 210-221. https://doi.org/10.18001/TRS.3.2.9

Northwestern Medicine. (2019). Dangers of Vaping. Northwestern Medicine. https://www.nm.org/healthbeat/healthy-tips/emotional-health/vaping-4-risks-for-kids

Prokhorov, A. V., Khalil, G. E., Foster, D. W., Marani, S. K., Guindani, M., Espada, J. P., ... Sussman, S. (2017). Testing the nicotine dependence measure mFTQ for adolescent smokers: A multinational investigation. American Journal on Addictions, 26(7), 689-696. https://doi.org/10.1111/ajad.12583

Raloff, J., \& Mole, B. (2015). Vaping may harm the lungs. Retrieved March 22, 2020, from https://www.sciencenewsforstudents.org/article/vaping-may-harm-lungs

Soneji, S., Yang, J., Knutzen, K. E., Moran, M. B., Tan, A. S. L., Sargent, J., \& Choi, K. (2018). Online Tobacco Marketing and Subsequent Tobacco Use. Pediatrics, 141(2). https://doi.org/10.1542/peds.2017-2927

Shmerling, R. H. (2019). Can vaping damage your lungs? What we do (and don't) know. Harvard Health Blog. https:/www.health.harvard.edu/blog/can-vaping-damage-your-lungs-what-we-do-and-dont-know-2019090417734.

Singh, T., Arrazola, R. A., Corey, C. G., Husten, C. G., Neff, L. J., Homa, D. M., \& King, B. A. (2016). Tobacco Use Among Middle and High School Students--United States, 2011-2015. MMWR. Morbidity and Mortality Weekly Report, 65(14), 361-367. https://doi.org/10.15585/mmwr.mm6514a1

Thakrar, P. D., Boyd, K. P., Swanson, C. P., Wideburg, E., \& Kumbhar, S. S. (2020). E-cigarette, or vaping, product use-associated lung injury in adolescents: a review of imaging features. Pediatric Radiology, 50(3), 338-344. doi: $10.1007 / \mathrm{s} 00247-019-04572-5$

The NIDA Blog Team. (2019). Why Is Nicotine So Addictive? Retrieved from https://teens.drugabuse.gov/blog/post/why-nicotine-so-addictive

Trauth, J. A., Seidler, F. J., \& Slotkin, T. A. (2000). Persistent and delayed behavioral changes after nicotine treatment in adolescent rats. Brain research, 880(1-2), 167-172.

Truth Initiative. (2019). E-cigarettes: Facts, stats and regulations. Retrieved from https://truthinitiative.org/researchresources/emerging-tobacco-products/e-cigarettes-facts-stats-and-regulations

University of Colorado Denver. (2019). Teen study reveals how schools influence e-cigarette use, outlines prevention strategies. ScienceDaily. Retrieved April 4, 2020 from www.sciencedaily.com/releases/2019/09/190930180956.htm

Yuan, M., Cross, S. J., Loughlin, S. E., \& Leslie, F. M. (2015). Nicotine and the adolescent brain. The Journal of Physiology, 593(16), 3397-3412. https://doi.org/10.1113/JP270492 